\title{
Targeting metastasis through the inhibition of interleukin 6 and 8
}

\author{
Hasini Jayatilaka*,1 \& Jude M Phillip ${ }^{2}$ \\ ${ }^{1}$ Department of Pediatrics, Bass Center for Childhood Cancer, Stanford University, Stanford, CA, 94305, USA \\ ${ }^{2}$ Department of Medicine, Division of Hematology \& Oncology, Weill Cornell Medicine, New York, NY, 10065, USA \\ *Author for correspondence: hasinij@stanford.edu
}

\author{
"Enhancing our ability to treat breast cancer with improved patient outcomes will require the \\ development of novel combination strategies that simultaneously target both tumor growth and \\ metastasis"
}

First draft submitted: 17 January 2019; Accepted for publication: 22 January 2019; Published online: 12 February 2019

Keywords: IL-6 • IL-8 • metastasis • synergistic signaling

Metastasis is a complex, multistep process involving the spread of cancer cells from a primary tumor to distal sites throughout the body via the circulatory or lymphatic systems [1]. Breast cancers typically arise from a host of genetic aberrations $[2,3]$ that influence both disease progression and the therapeutic approaches utilized by physicians to combat the disease [4]. With the exceptions of estrogen receptor (ER), HER2, phosphatidylinositol-4,5-bisphosphate 3-kinase catalytic subunit alpha, PIK3CA, and AKT1, validated oncogenic drivers of breast cancer remain elusive. Directly targeting metastasis is essential with regards to therapeutic interventions and could have major medical and societal implications, as mounting scientific evidence shows that metastasis accounts for $90 \%$ of the cancer-related deaths [5]. Although targeting metastasis itself may seem a daunting task scientifically and logistically, progressive improvements in our knowledge of the disease is providing novel and innovative approaches.

Many chemokines and cytokines (mainly interleukins) play critical roles in the metastatic process, from influencing epithelial to mesenchymal transition (EMT) [6] and facilitating the detachment of tumor cells from the primary tumor mass, to regulating cell migration [7], promoting seeding by circulating tumor cells (CTCs) [8] and stimulating proliferation [9]. Recent studies have demonstrated that interleukins cooperatively regulate aspects of metastasis. For instance, IL-6 and IL-8 co-regulate tumor cell proliferation and migration and the seeding of CTCs [10-13]. Since tumor cells rely on coordinated interactions with different cell populations within the microenvironment (both malignant and stromal cells) for fitness and survival during tumorigenesis [14], it is logical that they would also rely on the synergistic interplay of secreted proteins, particularly employing interleukins to successfully metastasize.

Tumor cells autonomously produce IL-6 and IL-8 which synergistically attracts CTCs and promotes the selfseeding of breast, colon and melanoma tumors [13]. Furthermore, these cytokines enhance tumor cell migration through cell-autonomous paracrine mechanisms driven in part by the increase in local cell density [10,11]. Interestingly, this signaling is unique to tumorigenic metastatic cells but not normal or non-metastatic cancer cells. IL-6 and IL-8 promote cell migration within collagen rich extracellular matrices through downstream signaling via WASF3 and Arp2/3 complex and increases the formation of dendritic protrusions. Furthermore, pharmacological inhibition of the binding of these interleukins to their cognate receptors using tocilizumab (a humanized monoclonal antibody that targets the IL- 6 receptor) and reparixin (a small molecule that targets the IL-8 receptor) decreases effective metastasis to the lungs, liver and lymph nodes in preclinical breast cancer models [10].

Breast cancers, particularly triple negative breast cancers (TNBCs), lacking the expression of the estrogen receptor, progesterone receptor (PR) and HER2, are largely treated with chemotherapeutics [15]. Paradoxically, studies suggest that chemotherapies currently used in the clinic for treating primary triple negative breast cancers may induce breast cancer metastasis [16]. Considering this, strategies that target both tumor growth and tumor-specific signals that trigger and/or promote migration may be more efficacious than chemotherapies alone, or targeted therapies that primarily inhibit cell growth.

Future Medicine 
Combination therapy is a promising strategy to combat the ails of monotherapy and realizing the goal of a bitherapeutic strategy to target both cell growth and cell migration. The amalgamation of two or more therapeutic agents working synergistically or additively typically provides superior clinical benefits through the effective reduction of drug resistance or enhanced efficacy via the targeting of complimentary cellular mechanisms [17]. For instance, the combination of standard chemotherapy regimens with bevacizumab significantly prolongs the survival of patients with metastatic cancers of the colorectal, breast and lung [18-20]. Considering the evidence that suggests that the synergistic signaling of IL-6 and IL-8 plays a critical role in the metastatic cascade [10-13], the simultaneous use of tocilizumab and reparixin with other anticancer therapeutics could greatly decrease tumor growth and metastatic capacity of breast cancer cells.

Enhancing our ability to treat breast cancer with improved patient outcomes will require the development of novel combination strategies that simultaneously target both tumor growth and metastasis. However, it is also critical that we develop our understanding of how malignant cells co-opt these long-range (mainly chemical) and short-range (mainly biophysical) signaling mechanisms within the tumor microenvironment.

\section{Open access}

This work is licensed under the Attribution-NonCommercial-NoDerivatives 4.0 Unported License. To view a copy of this license, visit http://creativecommons.org/licenses/by-nc-nd/4.0/

\section{Financial \& competing interests disclosure}

This work was supported by National Institute on Aging, U01AG060903 (JMP). The authors have no other relevant affiliations or financial involvement with any organization or entity with a financial interest in or financial conflict with the subject matter or materials discussed in the manuscript apart from those disclosed.

No writing assistance was utilized in the production of this manuscript.

\section{References}

1. Steeg PS. Tumor metastasis: mechanistic insights and clinical challenges. Nat. Med. 12(8), 895-904 (2006).

2. Vogelstein B, Kinzler KW. Cancer genes and the pathways they control. Nat Med. 10(8), 789-799 (2004).

3. McPherson K, Steel CM, Dixon JM. Breast cancer - epidemiology, risk factors, and genetics. BMJ 321(7261), 624-628 (2000).

4. Phillip JM, Zamponi N, Phillip MP. It's a numbers game - density-dependent MMP activity mediates cancer cell migration. Oncotarget 9(74), 33867-33868 (2018).

5. Chambers AF, Groom AC, MacDonald IC. Dissemination and growth of cancer cells in metastatic sites. Nat. Rev. Cancer 2(8), 563-572 (2002).

6. Sullivan NJ, Sasser AK, Axel AE et al. Interleukin-6 induces an epithelial-mesenchymal transition phenotype in human breast cancer cells. Oncogene 28(33), 2940-2947 (2009).

7. Yang Y, Cheon S, Jung MK et al. Interleukin-18 enhances breast cancer cell migration via down-regulation of claudin-12 and induction of the p38 MAPK pathway. Biochem. Biophys. Res. Commun. 459(3), 379-389 (2015).

8. Zhang Y, Ma Q, Liu T et al. Interleukin-6 suppression reduces tumour self-seeding by circulating tumour cells in a human osteosarcoma nude mouse model. Oncotarget 7(1), 446-458 (2016).

9. Roca H, Craig MJ, Ying C et al. IL-4 induces proliferation in prostate cancer PC3 cells under nutrient-depletion stress through the activation of the JNK-pathway and survivin up-regulation. J. Cell. Biochem. 113(5), 1569-1580 (2012).

10. Jayatilaka H, Tyle P, Chen JJ et al. Synergistic IL-6 and IL-8 paracrine signalling pathway infers a strategy to inhibit tumour cell migration. Nat. Commun. 8, 15584 (2017).

11. Jayatilaka H, Umanzor FG, Shah V et al. Tumor cell density regulates matrix metalloproteinases for enhanced migration. Oncotarget 9(66), 32556-32569 (2018).

12. Hartman ZC, Poage GM, Den Hollander P et al. Growth of triple-negative breast cancer cells relies upon coordinate autocrine expression of the proinflammatory cytokines IL-6 and IL-8. Cancer Res. 73(11), 3470-3480 (2013).

13. Kim MY, Oskarsson T, Acharyya S et al. Tumor self-seeding by circulating cancer cells. Cell 139(7), 1315-1326 (2009).

14. Tabassum DP, Polyak K. Tumorigenesis: it takes a village. Nat. Rev. Cancer 15(8), 473-483 (2015).

15. Isakoff SJ. Triple-negative breast cancer: role of specific chemotherapy agents. Cancer J. 16(1), 53-61 (2010).

16. Karagiannis GS, Pastoriza JM, Wang Y et al. Neoadjuvant chemotherapy induces breast cancer metastasis through a TMEM-mediated mechanism. Sci. Transl. Med. 9(397), eaan0026 (2017).

17. Mokhtari RB, Homayouni TS, Baluch N et al. Combination therapy in combating cancer. Oncotarget 8(23), 38022-38043 (2015). 
18. Miller KD, Chap LI, Holmes FA et al. Randomized Phase III trial of capecitabine compared with bevacizumab plus capecitabine in patients with previously treated metastatic breast cancer. J. Clin. Oncol. 23(4), 792-799 (2005).

19. Hurwitz H, Fehrenbacher L, Novotny W et al. Bevacizumab plus irinotecan, fluorouracil, and leucovorin for metastatic colorectal cancer. N. Engl. J. Med. 350(23), 2335-23342 (2004).

20. Adams GP, Weiner LM. Monoclonal antibody therapy of cancer. Nat. Biotechnol. 23(9), 1147-1157 (2005). 
